\title{
Detection of Insulation Flaws and Thermal Bridges in Insulated Truck Box Panels
}

\author{
by L. Lei*, A. Bortolin** ${ }^{*}$ P. Bison** and X. Maldague*
}

* University Laval, Quebec, Canada.

${ }^{*}$ CNR-ITC Padova, Italy.

\begin{abstract}
This paper focuses on the detection of defects and thermal bridges in insulated truck box panels, utilizing Infrared thermography. Unlike the traditional way in which passive thermography is applied, this research uses both heating and cooling methods in active thermography configurations. Lamp heating is used as the hot external stimulation, while a compressed air jet is applied as the cold external stimulation. An IR camera captures the whole process. In addition, numerical simulations under COMSOL platform are also conducted. Experimental and simulation results for two situations are compared and discussed.
\end{abstract}

\section{Introduction}

The increasing cost of energy has made energy saving a vital necessity in the current world. One of the examples involves, "Maintaining the cold chain": the correct transport of perishable foodstuffs in refrigerated vehicles, especially for dairy products, meat and frozen foods, which has become the key part of every successful distributor's food safety program. Therefore, a suitable thermal insulation implemented in refrigerated vehicles is essential for saving energy while maintaining an appropriate conservation of the foodstuffs. There are some agreements concerning thermal insulation tests which ensures the suitability for the transport of food in refrigerated conditions, for example ATP: "The Agreement on the Transport of Perishable foodstuffs" [1], which establishes standards for the international transport of perishable food between the states that ratify the treaty since 1970.

The Construction Technologies Institute of the Italian National Research Council (ITC- CNR), our collaborator, hosts a wide testing facility for refrigerated vehicles or insulated roll containers and it is also authorized by the Italian Ministry of Transport to perform such ATP tests [2][3]. The ATP standard test is a procedure that measures the insulating performance of truck panels with a global approach; however if there are some local flaws or thermal bridges inside the panels, which could not be measured by ATP tests, then the insulation and the stability of the temperature can no longer be guaranteed. Usually truck panels are manufactured from composite insulated materials in order to ensure that the interior of the truck is maintained at a cold temperature [4]. Therefore, this research attempts to detect the local flaws inside a truck panel specimen with a straightforward visualization.

\section{Sample \& Methods}
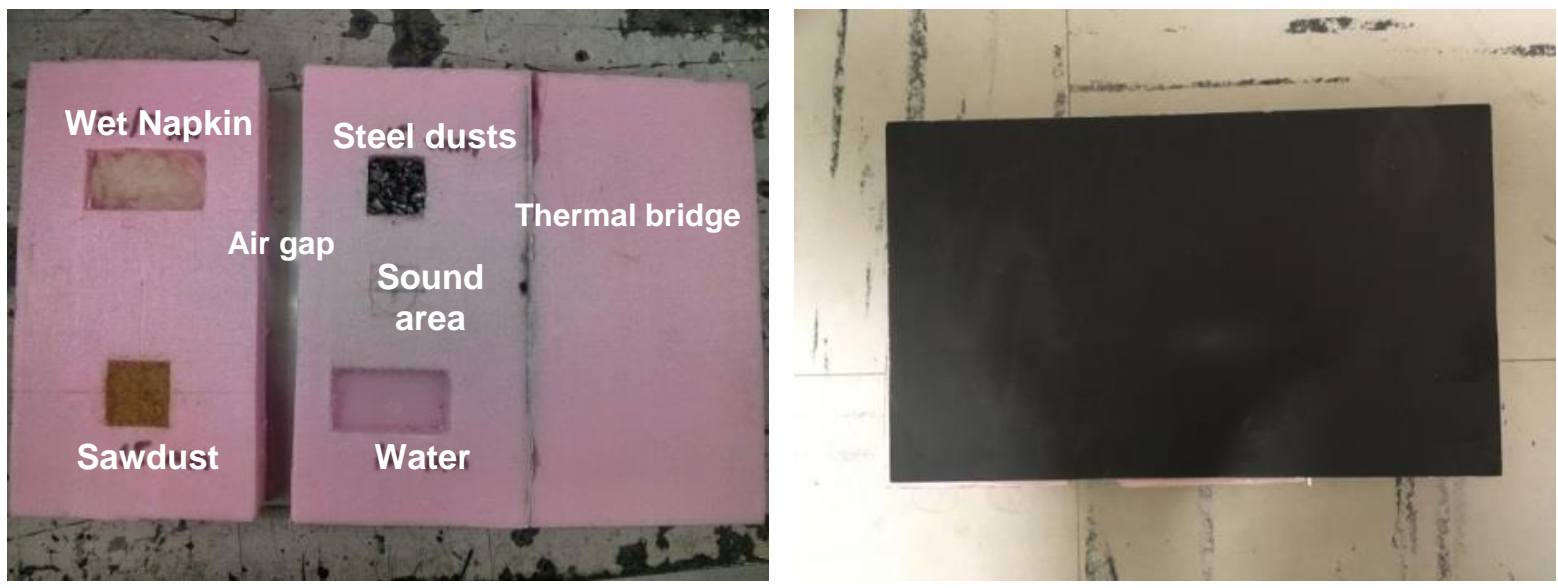

Fig.1. Details of defects inside the specimen (left) and final specimen to test (right)

In this research, several possible different types of defects inside a truck panel will be examined. A panel containing these defects has been constructed and is shown in Fig.1. The insulated material used is Polystyrene, inserted between two thin Aluminum plaques. A small Aluminum plaque is embedded 'vertically' inside acting as a thermal bridge. Other flaws are defined as an air gap, a defect involving a wet napkin, a hole filled with sawdust, another hole filled with steel dust and a defect filled with water; these are representative of current truck box fabrication and 
potential defects. The simulated panel was painted before the test to increase emissivity of aluminum. Particularly the thermal bridge and the defect of water are the main targets to identify, since they appear regularly in the gaps of truck panels. A sound area with no defects has also been defined for later comparison and reference, which is located at the center of the specimen. The specification details can be found in Table 1:

Table 1. Specimen specification details

\begin{tabular}{|l|c|c|c|c|}
\hline & Aluminum plaques & Foam & Air Gap & Thermal Bridge \\
\hline \multirow{3}{*}{ Dimensions $(\mathrm{mm})$} & $250 * 150 * 1$ & $250 * 150 * 25$ & $15^{*} 150 * 25$ & $1 * 150 * 25$ \\
\cline { 2 - 5 } & Wet Napkin & Sawdust & Steel dusts & Water \\
\cline { 2 - 5 } & $40 * 20 * 10$ & $20 * 20 * 15$ & $20 * 20 * 15$ & $40 * 20 * 10$ \\
\hline
\end{tabular}

In order to localize the possible defects inside the specimen, active Infrared Thermography [5, 6], one of the Non-destructive Testing \& Evaluation techniques, is applied for diagnostics. Basically, the specimen to inspect is thermally stimulated and the subsequent temperature evolution is recorded to reveal possible subsurface flaws. In this study two opposing approaches of external stimulations are then applied with the goal of clearly detecting the flaws in the truck panel. One approach uses a traditional lamp heating; the other method involves air cooling.

\section{Experimental set-up}

The experiment is set up with the following equipment, shown in Fig.2:

- Two halogen lamps each with heat source of 500W;

- A FLIR Phoenix thermal camera (640 × 512 pixels, InSb, 3-5 $\mu \mathrm{m})$;

- Compressed air connected by a tube and a nozzle with a diameter of $5 \mathrm{~mm}$ (Zoomed bottom left).

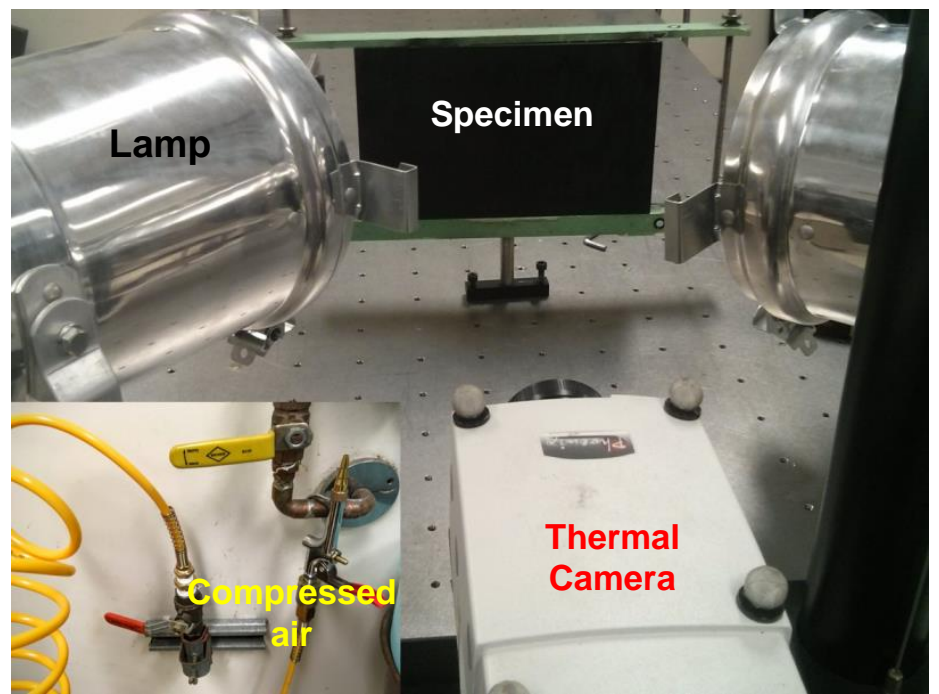

Fig. 2 Experimental Set-up

The whole procedure of study is described as follows:

For lamp heating, two halogen lamps each with a total heat source of $1000 \mathrm{~W}$ are employed at a distance of 0.4 $\mathrm{m}$ and at an angle of about $45^{\circ}$ for the purpose of homogeneously heating the surface. The duration of the heating process is only $20 \mathrm{~s}$.

For air-cooling, compressed air with a temperature of about $15^{\circ}$ is sprayed at a distance of $0.4 \mathrm{~m}$ and at an angle of about $45^{\circ}$ in the left front of the specimen's surface. In this case, contrary to the heating process, the specimen was preheated to about $30^{\circ} \mathrm{C}$, which is similar to the real situation for detection of truck panel defects in the summer. The duration of the cooling-down process is about 20s as well. It is noted that the compressed air has a pressure of 6 bar.

Both processes have been recorded by the thermal infrared camera with a resolution of $640 \times 512$ pixels. 


\section{Simulation models}

To obtain a comparative result, numerical models have also been developed, with the Finite Element Method under COMSOL Multiphysics $®$. In addition to the similar transient problem appearing in experimental conditions, static regime simulation is also taken into account. The influences of heat conduction, convection and radiation (surface-tosurface and surface to ambient) on the heat flow through the defect have been simulated and analyzed.

The physical nature of the heat transfer is governed by the differential equations such as the one of the heat transfer by conduction, convection and radiation with temperature dependant thermal properties of materials involved. The differential equation, governing pure conductive heat transfer, to be solved on the model domain is:

$$
\rho C_{p} \frac{\partial T}{\partial t}-\nabla \cdot(k \nabla T)=0
$$

where $\rho$ is the density $\left(\mathrm{kg} / \mathrm{m}^{3}\right), \quad C_{p}$ is the material heat capacity at constant pressure $(\mathrm{J} /(\mathrm{kg} \cdot \mathrm{K})), T$ is absolute temperature $(K)$ and $k$ is the material thermal conductivity $(W /(m \cdot K))$ and $t$ is the time.

The boundary condition included heat transfer by convection and radiation from the object surfaces and the heat source $q_{0}$ applied on the front surface as the following:

$$
n(k \nabla T)=q_{0}+h_{c v}\left(T_{a m b}-T\right)+\sigma \epsilon\left(T_{a m b}^{4}-T^{4}\right)
$$

where $h_{c v}$ is the constant onvective heat transfer coefficient, $\sigma$ is the Stefan-Boltzmann constant and $\varepsilon$ is the emissivity that is the ratio of radiant emittance of an object to that of a blackbody at the same temperature. Its value lies from zero for a non radiating object and 1.0 for a blackbody. $T_{a m b}$ is the Room temperature.

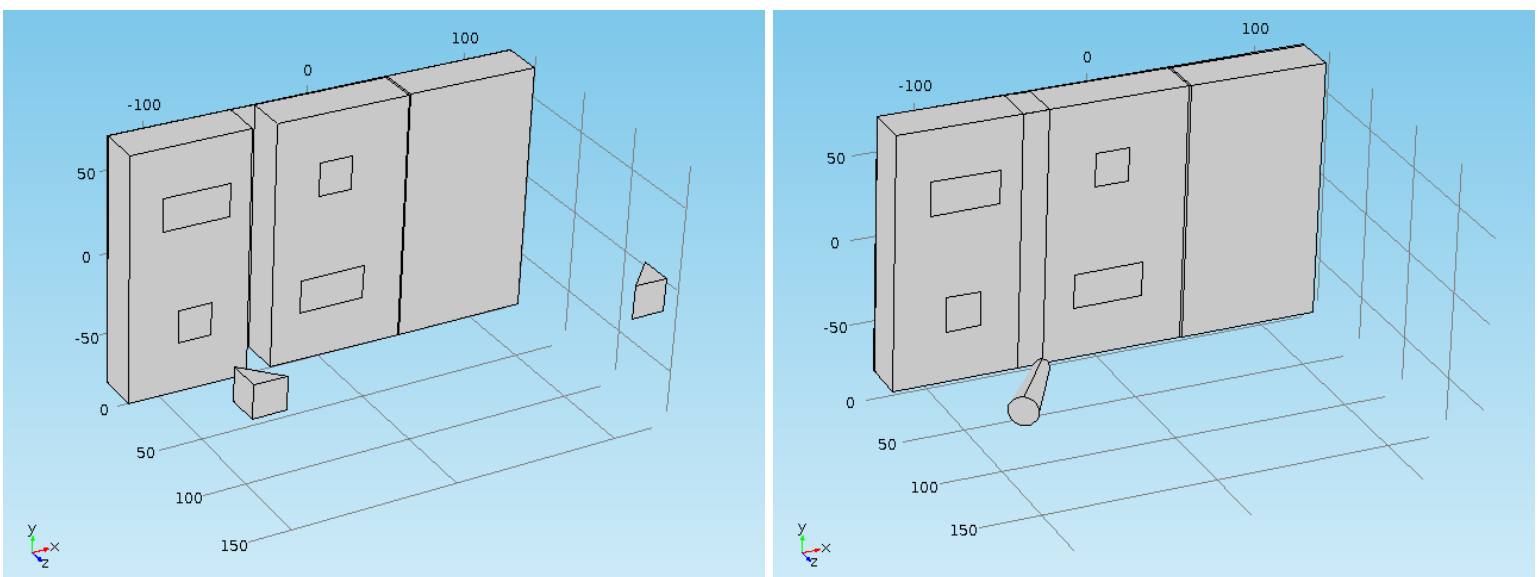

Fig. 3 Simulation 3D models view: Heating with two halogen lamps (Left) and Cooling with compressed air by a nozzle (Right)

Parameters for each model are introduced as follows:

For lamp heating, Heat Transfer in Solid with Surface-to-Surface Radiation module is applied in the model. Therefore, one assumes that there is no effect by convective transfer $\left[h_{c v}=0\right.$ in eq. (2)]

For air cooling, Heat transfer and Single Phase Laminar Flow modules are implemented since the convection effect in this case has a more significant influence. The Multiphysics setting is also set as a Non-Isothermal Flow. Then one assumes that there is no effect by the heat source $\left[q_{0}=0\right.$ in eq. (2)]

eq. (2)

For the static regime, a simple Heat Transfer in Solid module is employed. Thus one has $h_{c v}=0$ and $q_{0}=0$ in

All materials properties applied in the models can be found in Table. 2. 
Table 2. Materials properties

\begin{tabular}{|c|c|c|c|c|c|c|}
\hline & $\begin{array}{l}\text { Density } \\
\rho\left[\mathrm{Kg} / \mathrm{m}^{3}\right]\end{array}$ & $\begin{array}{c}\text { Thermal } \\
\text { conductivity } \\
\boldsymbol{k}[\mathbf{W} /(\mathbf{m} \cdot \mathbf{K})]\end{array}$ & $\begin{array}{c}\text { Heat } \\
\text { Capacity } \\
\boldsymbol{C p}_{\boldsymbol{p}} \\
{[\mathbf{J} /(\mathbf{K g} \cdot \mathbf{K})]}\end{array}$ & $\begin{array}{c}\text { Surface } \\
\text { Emissivity } \\
\boldsymbol{\varepsilon}\end{array}$ & $\begin{array}{c}\text { Dynamic } \\
\text { viscosity } \\
\boldsymbol{\mu}\left[10^{-5}\right. \\
\mathbf{k g} / \mathbf{m} \cdot \mathbf{s}]\end{array}$ & $\begin{array}{c}\text { Ratio of } \\
\text { specific } \\
\text { heat } \\
\mathbf{Y}\end{array}$ \\
\hline $\begin{array}{c}\text { Aluminum } \\
\text { (plaques and } \\
\text { Thermal bridge) }\end{array}$ & 2700 & 238 & 900 & 0.77 & & \\
\hline Foam & 24 & 0.03 & 1300 & & & \\
\hline Air & 1.20 & 0.024 & 1.005 & & 1.846 & 1.4 \\
\hline Sawdust & 192 & 0.08 & 900 & & & \\
\hline Water & 1000 & 0.58 & 4.180 & & & 1.33 \\
\hline Steel & 7850 & 44.5 & 475 & & & \\
\hline Lamps & 8700 & 400 & 10 & 0.99 & & \\
\hline
\end{tabular}

\section{Results and Discussion}
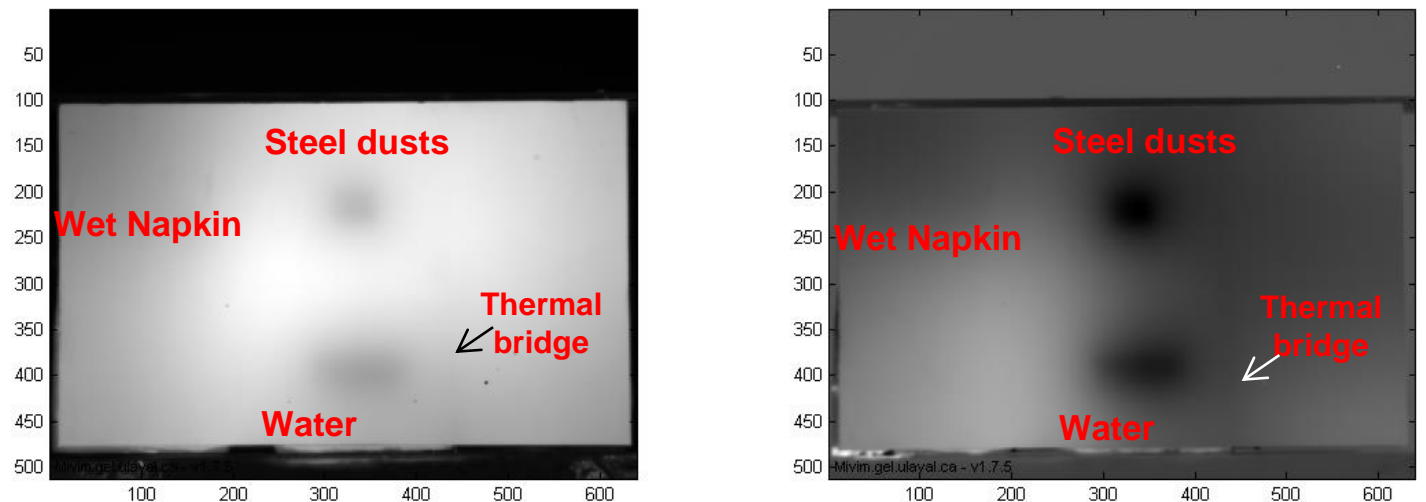

Fig. 4. Temperature distribution of panel surface with Lamp heating (left) and Air cooling (right) [Experiments]

Time $=7.5 \mathrm{~s}$ Temperature $(\operatorname{deg} \mathrm{C})$

$\Delta 23$

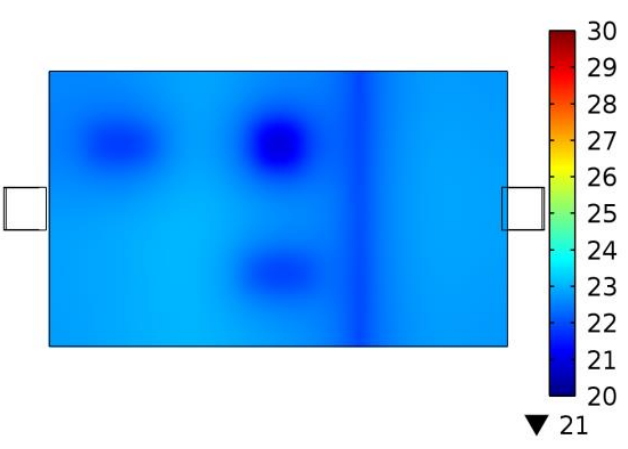

Time $=2 \mathrm{~s}$ Temperature $(\operatorname{deg} \mathrm{C})$

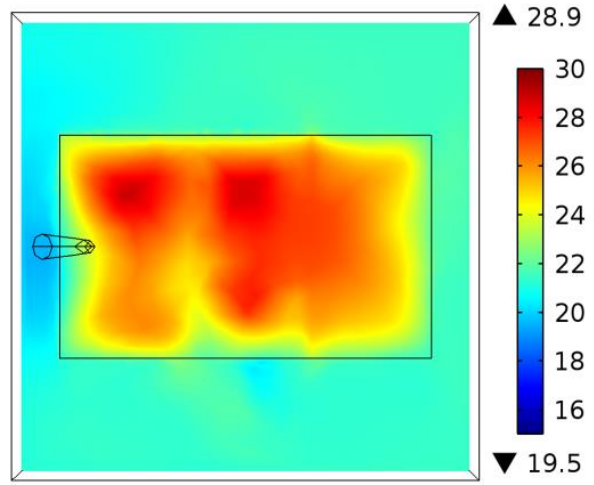

Fig. 5. Temperature distribution of panel surface with Lamp heating (left) and Air cooling (right) [Simulation]

Several image post-processing methods can be applied, such as Pulsed Phase Thermography [7], Principal Component Thermography [8]. PCT technique uses "singular value decomposition (SVD) to reduce the matrix of observations to a highly compact statistical representation of the spatial and temporal variations relating to contrast information associated with underlying structural flaws". Temperature distributions of the panel surface for both cases are presented in Fig. 4 and Fig. 5. Both lamp-heating and air-cooling images shown are the third image post-processed by 
the PCT. It is clear that in experimental results, the defects of metal and water are easy to identify, in both heating and cooling cases. The Thermal bridges are slightly clearer in the cooling process than the heating process. However, the defect of the wet napkin has almost not been displayed in both cases. On the other hand, in simulation results, due to the ideal conditions, one can clearly notice the four defects mentioned above. Figure 5 indicates that there is a more obvious view of defects in lamp heating as opposed to air cooling, the detection time of the latter is much less.

Nonetheless, in both experiments and simulation models the sawdust defect was impossible to detect, due to its thermal conductivity close to Polystyrene. The same situation occurred for the air gap, which could not be detected.

In addition to the straight views comparison in the thermal images, the quantitative computation has also been carried out at the same time. Temperature evolutions in time for both cases are illustrated in Fig. 6 and Fig. 7. Here one calculates the cases of the Water and Thermal Bridge (which are generally found in truck panels) compared to the Sound Area without defects. In both situations, experimental and simulation, it is observed that during lamp-heating, the temperatures of the thermal bridge and water defect area increased more slowly than the sound area value, while they decreased more slowly as well during air cooling. Quantitatively, for lamp heating, all defect temperature areas are lower than the sound area temperature. For air-cooling, on the contrary, all temperatures of defect areas are higher than that of the sound area (The descendent curve at the end of heating profile is due to the delay between the camera and lamps).
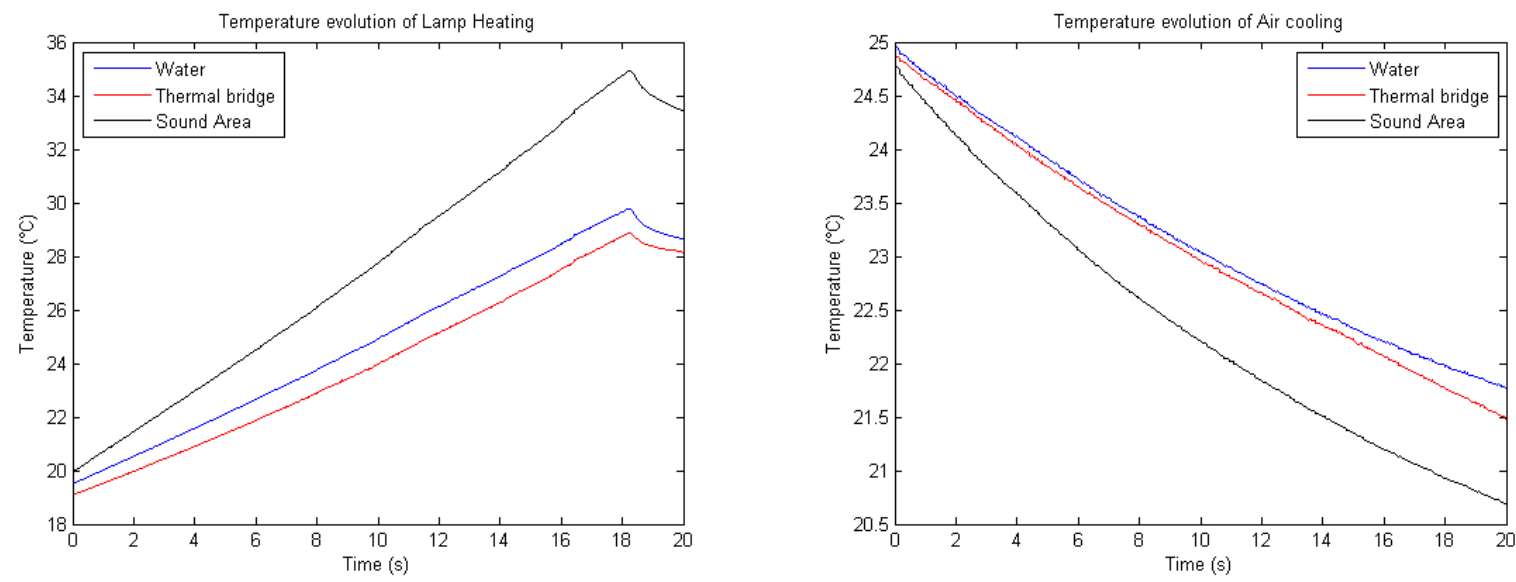

Fig. 6. Experimental Results of Temperature evolution with Lamp heating (left) and Air cooling (right)
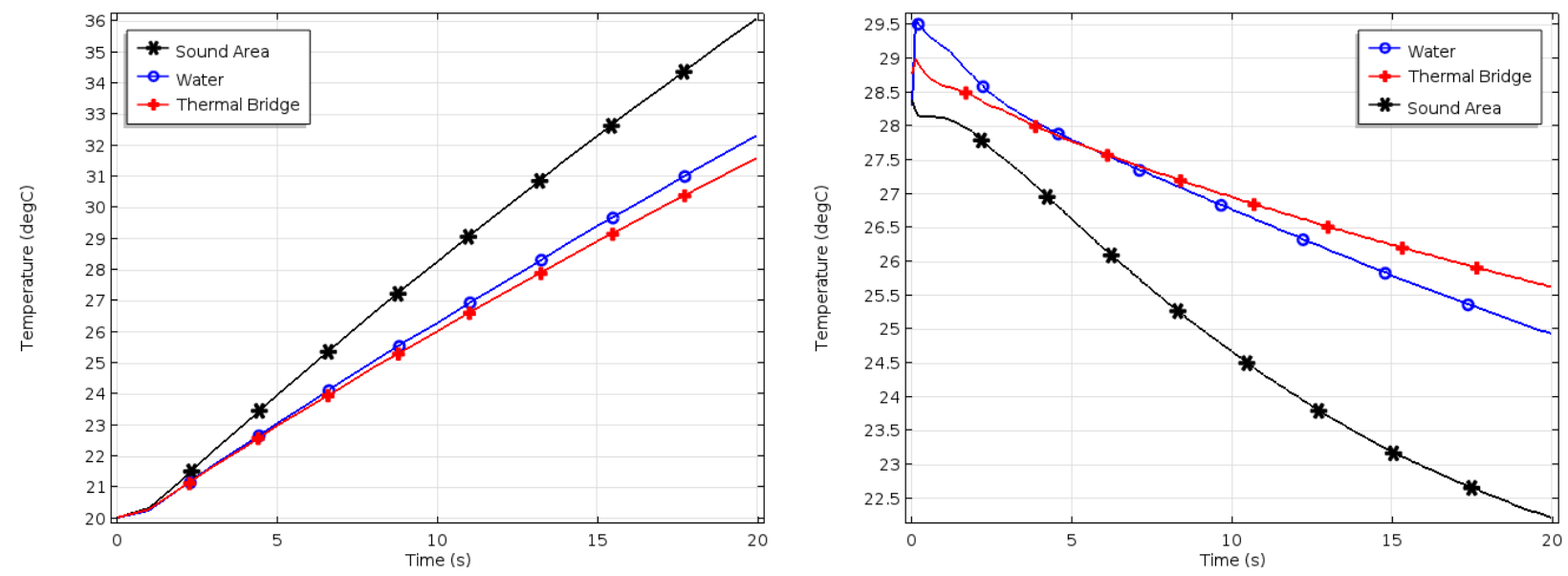

Fig. 7. Computational Results of Temperature evolution (Left: Lamp heating; Right: Air cooling)

From the results above, one calculates the thermal contrasts between two defects and the sound area. Moreover, the corresponding contrast peaks have been determined, shown in Table 3:

Table 3. Thermal contrast peak table

\begin{tabular}{|l|l|l|l|l|}
\hline & \multicolumn{2}{|c|}{ Experiment } & \multicolumn{2}{c|}{ Simulation } \\
\hline ROI & Heating & Cooling & Heating & Cooling \\
\hline Sound area & 0 & 0 & 0 & 0 \\
\hline Water & 5.17 & 1.08 & 3.80 & 1.6 \\
\hline Thermal bridge & 6.07 & 0.87 & 4.52 & 2.3 \\
\hline
\end{tabular}


This table indicates that for both cases, the Lamp Heating method has a higher contrast peak than the Air Cooling method. While comparing the temperature evolution profiles, the Air Cooling method requires less time to reach the contrast peak. This coincides with the detection of flaws in thermal images view.

For further exploration of the detection of each method, one defines here a "Threshold" with $C_{p}=1$ as the limit of detection, where $C_{p}$ is the peak of thermal contrast. As the experimental and computational results are very comparable from above, we change the size (diameter) of the water defect and the thickness of thermal bridge in both heating and cooling models to seek the limit of detection. For supplementary comparison, we add also a regular situation: the static regime. This acts as the traditional passive thermography. One imagines that the truck is under sunshine in the exterior during summer, and inside temperature is kept as the cold chain, which should be $-20^{\circ} \mathrm{C}$. Then an infrared camera from outside observes the truck panel. Different sizes of water defects and widths of the thermal bridge have been performed during the same period (20s) for this case.

All the computational results are presented in the following figures:
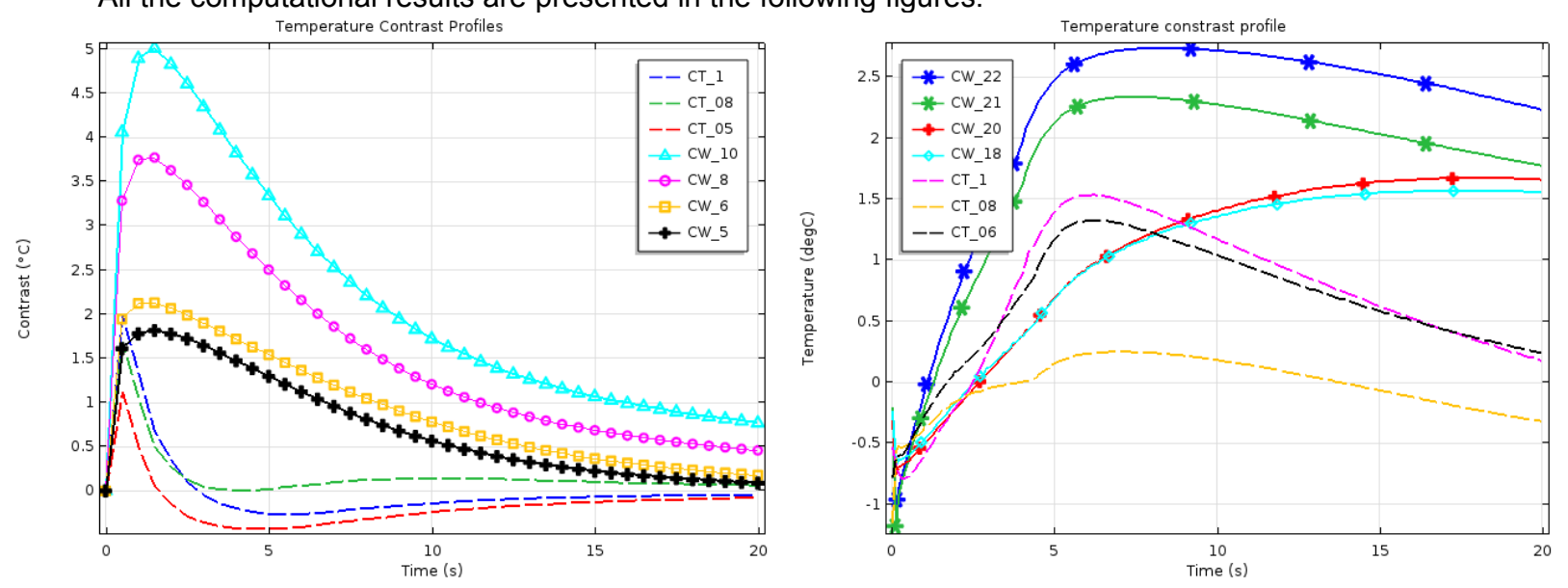

Fig. 8. Temperature contrast profiles for models (Left: static regime; Right: Air cooling)
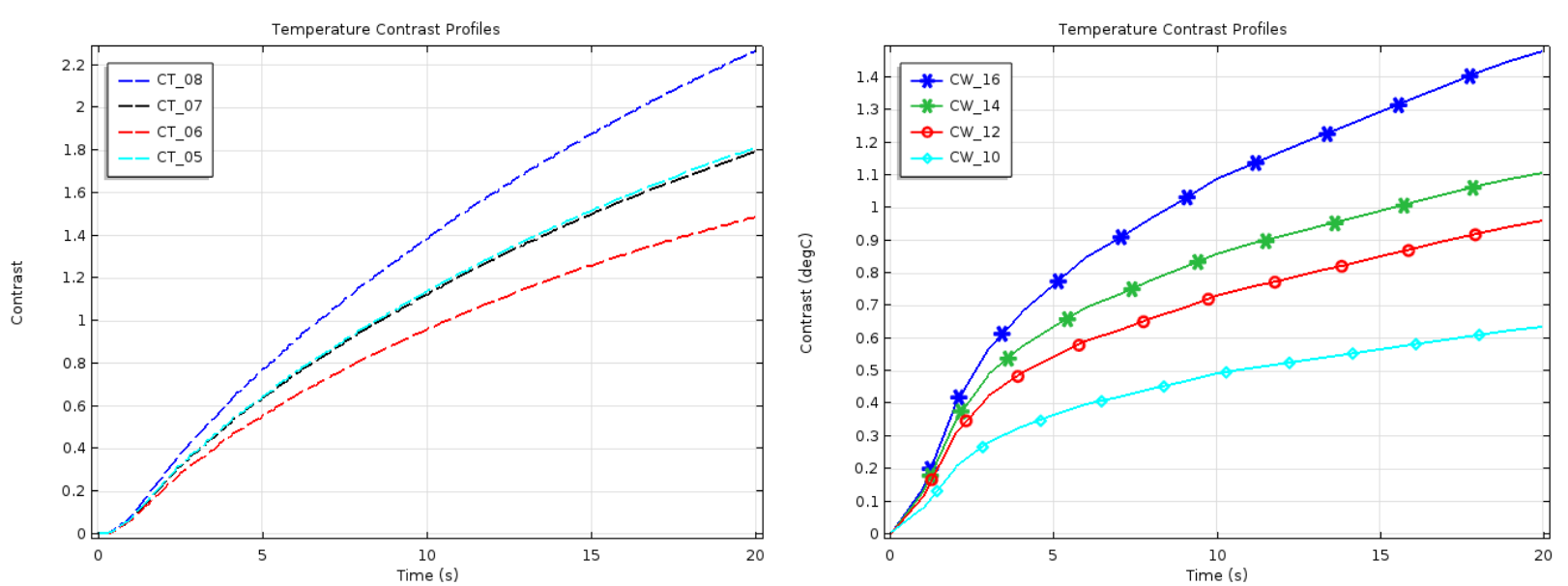

Fig. 9. Temperature contrast profiles for Lamp heating model (Left: thermal bridge, right: water defects)

Where $C T$ represents the contrast of the thermal bridge, $C W$ represents the contrast of the water defect in all figures, and the numbers indicate the diameter and the thickness respectively.

From the results above, it is evident that the static regime and lamp heating have more consistent profiles for the two types of flaws. All profiles in static regime increased rapidly at the beginning, as a consequence of temperature difference between sound area and defects area. Soon they all reached at a peak value subsequently. The contrast peak values varied according to different sizes of defect. Evidently, the contrast values of water defects are more sizable than that of thermal bridge. Then all profiles decreased gradually since the propagation of the heat inside the panel. In the end, they tend around whole uniform.

While in air-cooling case there are several non-uniform profiles, nonetheless, the contrast profiles of water defect with large sizes $(21 \mathrm{~mm}$ and $22 \mathrm{~mm}$ ) and those of thermal bridge with all sizes have the similar tendency. They increased at the beginning due to the convection by air and then decreased slowly because of the propagation of heat dissipation inside the panel. However, for the contrast profiles of water defects with smaller sizes $(20 \mathrm{~mm}$ and $18 \mathrm{~mm})$, 
they appeared as a unique increase. They might decrease again for long time modeling. An unusual thing found in aircooling model was the thermal bridge with $0.8 \mathrm{~mm}$ thick had a lower contrast than those of $1 \mathrm{~mm}$ and $0.6 \mathrm{~mm}$, this might be due to the influence of disturbance by airflow.

For lamp-heating situation, one can observe that whole contrast profiles had a uniform tendency, which they all raised with time. This is as a result by radiation heating.

Finally, according to our "Threshold" $C_{p}=1$, then from the figures the limit of detection of these three cases is (during a period of time [20s]):

- Static regime: Water defect diameter $5 \mathrm{~mm}$; Thermal Bridge Thickness $0.5 \mathrm{~mm}$.

- Air cooing: Water defect diameter $18 \mathrm{~mm}$; Thermal Bridge Thickness $0.6 \mathrm{~mm}$.

- Lamp heating: Water defect diameter $14 \mathrm{~mm}$; Thermal Bridge Thickness $0.5 \mathrm{~mm}$.

One must note that, these results have been obtained by simulation models, which were undertaken in ideal situations. The results might be different in the real case.

\section{Conclusion}

This study concentrates on the detection of defects and thermal bridges in insulated truck box panels, by active Infrared thermography. Comparison between heating and cooling approaches for experiments and models has been established. In addition, passive thermography detection in computational models has been presented. Results demonstrate that the compressed air spray is more rapid than the traditional heating method in providing successful detection. Even if the traditional heating approach provides clearer results, in reality it is not easy and practical to heat a whole truck box to conduct inspection: the compressed air spray approach is much more convenient.

A consideration of replacing compressed air by liquid nitrogen will be investigated in future work.

\section{Acknowledgment}

This research was supported by the governments of Italy and Quebec, and by the Natural Sciences and Engineering Research Council of Canada (NSERC). We are also thankful to our collaborative institute CNR-ITC Padova which provided expertise that greatly helped in this research.

\section{REFERENCES}

[1] ATP - UN-ECE, Agreement Transport Perishables. Agreement on the international carriage of perishable foodstuffs and on the special equipment to be used for such carriage, Geneva 1970.

[2] Tassou S.A., De-Lille G., Ge Y.T. 2009, Food transport refrigeration - Approaches to reduce energy consumption and environmental impacts of road transport, Applied Thermal Engineering, Volume 29, Issues 8 9, Pages 1467-1477.

[3] Dragan A., Rossi S., Bison P., Panozzo G. 2009, Experimental evaluation of the optical absorption coefficient of a sandwich panel for insulated vehicles. Quantitative InfraRed Thermography Journal, 6(2):207-223.

[4] Bortolin A., Bison P., Cadelano G., Ferrarini G., Lei L. and Maldague X. 2015, Mapping the heat flux of an insulated small container by infrared thermography, 13th Advanced Infrared Technology and Applications (AITA13), Pisa, Italy.

[5] Maldague X. 2001, Theory and practice of infrared technology for nondestructive testing, Wiley.

[6] D. Balageas, X. Maldague, D. Burleigh, V.P. Vavilov, B. Oswald-Tranta, J.-M. Roche, C. Pradere, G.M. Carlomagno, "Thermal (IR) and Other NDT Techniques for Improved Material Inspection," J. of NDE, 35: 18, March 2016.

[7] X. Maldague, S. Marinetti, "Pulse Phase Infrared Thermography," J. Appl. Phys, 79[5]: 2694-2698, 1 Mar. 1996.

[8] Rajic N. Principal component thermography [R]. Defence Science And Technology Organisation Victoria (AUSTRALIA) Aeronautical And Maritime Research Lab, 2002. 